\title{
ISOMORPHIC CONGRUENCE GROUPS AND HECKE OPERATORS
}

\author{
by R. A. RANKIN
}

(Received 15 October, 1965)

Let $G, H, K$ be groups such that $G$ is normal in $K$ and $G \subseteq H \subseteq K$. Let $I(H, K)$ be the set of inner automorphisms of $K$ restricted to $H$; thus $\alpha \in I(H, K)$ if and only if, for some $k \in K, \alpha(h)=k^{-1} h k$ for all $h \in H$. Let $\phi$ be an isomorphism of $H / G$ onto a subgroup $H^{(\phi)} / G$ of $K / G$. An isomorphism $\Phi$ of $H$ onto $H^{(\phi)}$ is called an extension of $\phi$ if

$$
\Phi(h) G=\phi(h G) \text { for all } h \in H .
$$

Such an extension need not exist in general, nor need the groups $H$ and $H^{(\phi)}$ be isomorphic.

Suppose that such an extension $\Phi$ does exist and that $\Phi \in I(H, K)$, so that, as above, $\Phi(h)=k^{-1} h k$. Since $\phi(h G)=\Phi(h) G=k^{-1} h k G=k^{-1} h G k$, it follows that $\phi \in I(H / G, K / G)$.

Let $N$ be a positive integer and put

$$
I=\left[\begin{array}{ll}
1 & 0 \\
0 & 1
\end{array}\right], \quad U=\left[\begin{array}{ll}
1 & 1 \\
0 & 1
\end{array}\right], \quad T=\left[\begin{array}{ll}
a & b \\
c & d
\end{array}\right] .
$$

Let $\Omega$ and $\Omega_{N}$ be the sets of all matrices $T$ with entries in $Z$, the ring of all integers, and $Z_{N}$, the ring of residues modulo $N$, respectively. Put

$$
\begin{aligned}
& \Gamma^{*}(1)=\{T: T \in \Omega, \operatorname{det} T= \pm 1\}, \quad \Gamma(1)=\{T: T \in \Omega, \operatorname{det} T=1\}, \\
& \Gamma(N)=\{T: T \in \Gamma(1), T \equiv I(\bmod N)\}, \quad K_{N}=\left\{T: T \in \Omega_{N},(\operatorname{det} T, N)=1\right\} .
\end{aligned}
$$

Then $K_{N}$ contains normal subgroups $G_{N} \sim \Gamma(1) / \Gamma(N), G_{N}^{*} \sim \Gamma^{*}(1) / \Gamma(N)$ arising from the natural homomorphism $\omega: \Omega \rightarrow \Omega_{N}$.

Let $\Gamma$ be a subgroup of $\Gamma^{*}(1)$ containing $\Gamma(N)$, and put $H_{N}=\omega(\Gamma) \sim \Gamma / \Gamma(N)$. In the theory of Hecke $T_{n}$-operators (see my forthcoming paper in Math. Annalen) one encounters isomorphisms $\phi \in I\left(H_{N}, K_{N}\right)$, where $\phi(h)=k^{-1} h k$ for some $k \in K_{N}$ and all $h \in H_{N}$, and det $k=n$ with $(n, N)=1$. It is of interest to know for what residues $n$ modulo $N$ the isomorphism $\phi$ has an extension $\Phi \in I\left(\Gamma, \Gamma^{*}(1)\right)$. The above remarks show that this can occur only if $\phi \in I\left(H_{N}, G_{N}^{*}\right)$, so that we must have $k^{-1} h k=g^{-1} h g$ for some $g \in G_{N}^{*}$ and all $h \in H_{N}$.

A case of particular interest arises when $L^{-1} U^{r} L \in \Gamma$ for some positive divisor $r$ of $N(r<N)$ and some $L \in \Gamma(1)$. Then, for some $T \in \Omega$ with $\operatorname{det} T=n$ (or $-n$ ), we must have $T^{-1} U^{r} T \equiv U^{r}(\bmod N)$. It follows easily from this that $n$ (or $\left.-n\right)$ must be congruent to a square modulo $N / r$.

As a kind of converse of this we show that, if $n \equiv v^{2}$ or $-n \equiv v^{2}(\bmod N)$, then $\phi$ always has an extension $\Phi \in I\left(\Gamma, \Gamma^{*}(1)\right)$. For we can take $A \in \Omega$ such that $\omega(A)=k$, and we then put $\Phi(S)=A_{n}^{-1} S A_{n}(S \in \Gamma)$, where $A_{n} \in \Gamma^{*}(1)$ and $v A_{n} \equiv A(\bmod N)$. In particular, this is possible for every $n$ prime to $N$, when $N=1,2,4, p$ or $2 p$, where $p$ is a prime and $p \equiv 3(\bmod 4)$. [This paper arises from an editorial observation that $167 \equiv 3(\bmod 4)$.

If we demand that $\Phi \in I(\Gamma, \Gamma(1))$, similar arguments apply, except that references to $-n$ are to be omitted.

UNIVERSITY OF GLASGOW

GlasGow, W. 2 